\title{
DÉBAT ET POLÉMIQUE DANS L'HISTOIRE DES MÉDIAS
}

\author{
Gabriel Thoveron ${ }^{1}$
}

Sous l'Ancien Régime, le droit d'être informé et celui d'informer n'appartiennent qu'aux Princes. Ils en usent et abusent, et, dès avant l'apparition des premières gazettes périodiques, utilisent les feuilles volantes, occasionnelles, pour servir leurs desseins. Ainsi "sous Louis XII, sous François Ier, le petit livret deviendra un instrument régulier de polémique internationale (...) la guerre à coups d'épée se complétera d'une guerre à coups de libelles" 2 . Les Du Bellay se font propagandistes de François Ier contre Charles Quint, et l'Arétin, vendant ses services tantôt à l'un, tantôt à l'autre de ces monarques, semble l'inventeur de la presse de chantage. Pendant la Révolution française, un ancien collaborateur des royalistes Actes des Apôtres rédige à Londres, en français, un journal, L'Ambigu, destiné aux immigrés ; il est interdit en France où cependant parviennent quelques exemplaires. Bonaparte, Premier Consul, riposte avec L'Argus, publié en anglais à Paris, destiné aux "républicains britanniques et aux Irlandais immigrés en France", en fait largement distribué dans les colonies de la

1 Professeur émérite à l'Université Libre de Bruxelles.

2 H. HAuser, Les sources de l'histoire de France, tome 1, Paris, 1906, cité par M. VARIN D'AINVELLE, La presse en France, Paris, PUF, 1965, p. 40.

Recherches en communication, $\mathrm{n}^{\circ} 20$ (2003). 
Couronne. Les deux publications sont violentes, car le ton est très vif des deux côtés de la Manche.

Les polémiques entre partisans et adversaires de la guerre avec l'Irak avaient de qui tenir : si la guerre, comme disait Clausewitz, est la continuation de la politique par d'autres moyens, il est normal qu'elle use des moyens de celle-ci. La radio, outil sans frontière, a joué un rôle considérable dans la guerre froide ${ }^{1}$, et continue même aujourd'hui dans des conflits larvés localisés, comme entre Cuba et les États-Unis.

À l'intérieur des monarchies de droit divin, les instruments de la lutte opposant réformés et catholiques ne peuvent être que des feuilles occasionnelles, circulant sous le manteau, ou des affiches -à l'image de celles illustrant, en 1517, les 95 thèses de Martin Lüther, à Wittenberg, ou de celles qui provoquent l'affaire des Placards, en France, en 1534. En dehors des périodes de crise, où le pouvoir se délite, comme, en France durant la Fronde (quand fleurissent des mazarinades dénonçant le Cardinal Ministre), toute critique religieuse ou politique est contrainte à la clandestinité, et circule sous le manteau sans qu'on puisse toujours la faire taire. Ainsi, de 1713 à 1793, les Nouvelles ecclésiastiques circulent de main en main, menant la lutte des jansénistes contre les jésuites, allant jusqu'à publier une gravure où Jésus, au désert, est tenté par un démon jésuite.

Mais "l'esprit de liberté, chassé de la presse politique, se réfugie dans la presse littéraire". En France, ce sont sans doute l'abbé Desfontaines et Fréron qui inaugurent, selon les termes de Georges Weill, "le journalisme critique, militant, agressif ", respectivement dans les Observations sur les écrits modernes (que le roi supprima d'ailleurs, en 1743, sur plainte de l'Académie française) et L'Année littéraire, sans cesse menacée dans une existence qui dura tout de même de 1754 à $1776^{2}$. "C'est dans la lie du XVIIIe siècle", écrira Delisle de Sales dans son méchant Essai sur le journalisme de 1735 à 1800 , "qu'il faut chercher l'époque tristement mémorable où s'établit la séparation entre ce que je serais tenté d'appeler les journaux à ambroisie et les journaux à venin". Le genre se définirait par "le besoin de déraisonner réuni au besoin de nuire", la critique exprimant

1 Voir par exemple l'ouvrage de J. SEMELIN, La liberté au bout des ondes, Paris, Belfond, 1997 ou de Fr. DonNAY, Radio-ingérence internationale, Bruxelles, Les dossiers du GRIP, $n^{\circ}$ 184-185, 1993.

2 G. WEILL, Le Journal. Origines, évolution et rôle de la presse périodique, Paris, 1934, pp. 92-93. 
"l' antipathie des gens qui vivent des feuilles contre ceux qui vivent de renommée".

La politique se dissimule sous la littérature. Sous le Consulat, dans le Journal des débats, l'abbé Julien Louis Geoffroy invente le feuilleton, chronique sise au rez-de-chaussée de la Une : il le consacre en principe à la critique théâtrale, mais l'histoire, la philosophie, la morale et même la politique y trouvent leur place. Il s'agit finalement de dérouler le fil d'un pamphlet permanent contre la Révolution, qu'il n'identifie qu'à la Terreur ${ }^{2}$.

\section{La presse artisanale participe au débat}

Les grandes révolutions du XVIIIème siècle proclament la liberté de la presse. Elle permettent, l'aube des démocraties, le développement des échanges politiques. L'idéal démocratique est ce que Gilles Achache, s'efforçant d'identifier les types de la persuasion politique, appelle modèle dialogique, "en ce que le dialogue au double sens d'échange de paroles et de rationalité à plusieurs, y est présenté comme la forme légitime" des rapports dans une démocratie. Les acteurs y sont des individus raisonneurs, "rationnels, libres et égaux", l'espace public est le "lieu de la détermination de l'intérêt général", du bien commun, la communication s'établit par "les médias discursifs" -comme le journal d'opinion- médias qui préfèrent "le discours à l'image". Ce modèle "s'impose à nous comme étant à la fois le plus ancien et celui qui est doté de la légitimité la plus assurée"3.

Un modèle idéal, car dans les faits, le débat devient vite passionné plutôt que rationnel, et tend à devenir combat.

Très vite, la presse d'opinion ne participe plus qu'à un dialogue de sourds. On n'y échange pas vraiment des arguments avec l'adversaire, on donne de la redondance à des opinions partagées par les lecteurs dont on entretient la foi, les passions.

Balzac, en 1842, en décrit le fonctionnement: "On appelle premier-Paris (on dirait aujourd'hui éditorial) la tartine qui doit se

1 Delisle De SAlES, Essai sur le journalisme depuis 1735 jusqu'à l'an 1800, Paris, 1811, p. 45.

2 G. WEILL, op. cit., pp. 134-135.

3 G. ACHACHE, "Le marketing politique", Hermès, Paris, CNRS, n 4, 1989, pp. 103 112. 
trouver en tête d'une feuille publique, tous les jours (...) Quoi que fasse le gouvernement, le rédacteur des premiers-Paris de l'opposition doit y trouver à redire, à blâmer, à gourmander, à conseiller. Quoi que fasse le gouvernement, le rédacteur des premiers-Paris ministériels est tenu de le défendre. L'un est une constante négation, l'autre une constante affirmation, en mettant à part la couleur qui nuance la prose de chaque parti (...) Ces faiseurs de tartines s'ingénient à n'être que la toile sur laquelle se peignent, comme des ombres chinoises, les idées de leur abonné. À chaque événement, l'abonné se forme une opinion et s'endort en se disant :«Je verrai demain ce que dira là-dessus mon journal». Le premier-Paris, qui n'existe que par la divination perpétuelle des pensées de son abonné, le surprend le lendemain agréablement en lui planifiant sa pensée"1.

La gazette politique joue alors rôle d'appartenance -au sens où l'entendait Jean Stoetzel - un rôle de reliance-au sens où l'entendait Roger Clausse, un rôle d'appartenance politique précise Madeleine Varin d'Ainvelle: "Le choix de tel journal distingue (le lecteur) des autres, le singularise en quelque sorte, tout en l'unissant au groupe des lecteurs de ce journal. Il se passe ici de véritables phénomènes de groupe : intégration, influences réciproques journal-lecteur, cristallisation des attitudes, recherche de modèles, cohésion, jeu de miroir : le journal renvoyant au lecteur sa propre image politisée; prise de conscience : l'expression par un autre de ce que l'on pense implicitement permettant de le penser d'une manière plus précise et plus affirmative"2. Évoquant la presse du temps de Louis-Philippe, Émile Zola observe qu'alors le journal n'existait que comme porte-parole : "on s'y abonnait par sympathie et l'abonné avait foi en lui. Il y avait un lien étroit entre les lecteurs et le rédacteur. Les listes des abonnés correspondaient en quelque sorte aux cadres du parti représenté par le journal. On ne saurait s'imaginer la solidité que lui donnait cette communauté de conviction"3.

Le journal d'opinion ne fabrique pas une opinion, il l'entretient. Dans ses colonnes, le lecteur ne découvre les arguments de l'adversaire qu'à travers les réponses qui lui sont apportées; il est confiant

1 Je cite d'après l'édition 1965, La presse parisienne, Paris, Pauvert, coll. "Libertés", 1965, pp. 42-44.

2 M. VARIN D'A AnVELLE, op. cit., p. 182.

3 E. Zola, "La presse parisienne", in Études de presse, Paris, vol. VIII, no 15-16, 1956, p. 262 (traduction d'un article publié par une revue russe dont l'écrivain était correspondant). 
que ses réponses sont au fond les siennes et que l'éditorialiste est son interprète. Le journal d'opinion lui dit ce qu'il pense, ou même souvent ce qu'il doit penser s'il veut continuer à être en accord avec le groupe politique dont il partage les idées.

Le lien est sans doute d'autant plus ferme si le groupe se réduit à un clan. Au début du $\mathrm{XX}^{\mathrm{e}}$ siècle, à Paris, "les nuances qui séparaient L'Action (...) de La Lanterne (...), celles qui faisaient de L'Aurore la concurrente du Radical, échappent, de nos jours, au point de vue doctrinal, à toute explication. Elles semblent avoir été de pure forme. Elles traduisaient des différences de tempérament et de styles"'. Ou, peut-être, elles servent de porte-parole à hommes politiques différents, même s'ils se situent dans un même parti : le journal est un outil du parlementarisme dans la mesure où il sert de courroie de liaison tantôt d'un groupe, tantôt simplement d'un élu, avec les citoyens électeurs.

S'adressant à un public bourgeois que le sentiment d'être détenteur naturel du pouvoir contribue à fortement politiser, la presse d'opinion attise les passions. Elle est violente. Elle peut l'être avec éloquence, notamment en France, où la gazette d'opinion oscille entre littérature et politique, cherchant souvent à compenser l'audace du fond par la qualité de la forme. Le genre entre dans l'histoire des Lettres, illustré par de grands noms, Paul-Louis Courrier à l'assaut des Bourbons, Hugo contre Napoléon le petit, Zola à la tête des dreyfusards. Tous ne s'exprimaient pas dans la presse, mais c'est en tête du premier numéro de La Lanterne que Rochefort lance son fameux "La France a trente-six millions de sujets, sans compter les sujets de mécontentement" et dans L'Aurore du 13 janvier 1898 que paraît le J'accuse de Zola.

La littérature n'exclut pas les insultes, celles de Léon Daudet, traitant, entre autres, Clemenceau, dans La Libre Parole, de "tête de mort sculptée dans un calcul biliaire", celles de Clemenceau qui voyait en Poincaré "une âme de lapin dans une peau de tambour", après celles d'un Louis Veuillot, qui dénonce la brutalité de certaines attaques, mais n'hésite pas y recourir. Le grand polémiste catholique, assure que "Le poignard le plus aigu, le poison le plus actif et le plus durable, c'est la plume dans les mains sales. Avec cela, on gâte un peuple, on gâte un siècle"2 ; mais il pense, lui, avoir les mains propres et ne se gêne pas pour couvrir ses adversaires d'injures (brute, fripon,

I R. Manevy, Histoire de la presse 1914-1939, Paris, Corréa, 1945, p. 8.

2 Cité par St. Brabant, La presse en citations, Bruxelles, AEEF, 2002, p. 39. 
vermine, eunuque, coquin, canaille, gredin, cuistre, goujat...), considérant que la charité est "quelquefois une vertu détestable". Le Patriote (qui deviendra Libre Belgique) pense de même, qui, par exemple, le 8 septembre 1884, traîne dans la boue le bourgmestre bruxellois Charles Buls : "Il ne se peut pas que l'exercice d'un droit constitutionnel dépende de la volonté d'un eunuque ou d'un traître, que des héros de brasseries ou de cabarets démontrent au pays au moyen de taches de sang répandues sur les trottoirs de nos boulevards, qu'il y a des sauvages ailleurs qu'au Congo et qu'il est permis d'assommer des citoyens à Bruxelles en plein midi"'.

Évoquant la violence des luttes de partis en Belgique à la fin du régime censitaire, alors que "de part et d'autre, la presse déverse l'injure ou la calomnie", Henri Pirenne observe que "poussée à ce point d'exaspération, la passion politique eût infailliblement abouti à la guerre civile si la restriction du droit de suffrage ne l'avait circonscrite aux limites de la bourgeoisie censitaire (...) le «pays légal» ne comprenait qu'une petite minorité de la nation et ses fureurs ne rencontraient que le calme ou l'indifférence de la masse"2. Le régime censitaire avait pour prétexte de réserver la participation politique à des citoyens que leurs revenus auraient rendu libres, indépendants, et essentiellement attachés à l'intérêt général, au bien commun ; mais son seul avantage semble avoir été de limiter les débordements de la fureur politique en réduisant le nombre de ceux dont elle s'emparait.

À partir du moment où le droit de vote se généralise, et touche enfin les groupes les moins favorisés de la société, lorsque par exemple, en Belgique, le mouvement socialiste vient troubler le face à face traditionnel des bourgeois catholiques et des libéraux, l'affiche entre dans le débat aux côtés du journal. Le Parti Ouvrier sera le premier, dès sa fondation en 1885 , à utiliser couramment des placards comme moyen de propagation de ses idées, placards faits d'abord seulement de texte, puis, environ dix ans plus tard, illustrés, et bientôt en couleurs ${ }^{3}$. Les autres partis sont naturellement conduits à le suivre, et le débat se déploie dans les rues, sur les murs.

1 P. STÉPHANY, Histoire d'un journal libie, Louvain-la-Neuve, Duculot, 1996, p. 38.

2 H. PIRENnE, Histoire de la Belgique, tome 4, Bruxelles, La Renaissance du Livre, 1952, p. 143.

3 L. Flagothier-Musin et R. FlagothiER, 75 ans de luttes sociales à travers l'affiche, Liège, 1981, pp.7-8. On suivra l'évolution dans MABILLE, 
L'affiche, comme la presse, argumente. Elle comporte du texte, mais il se mêle à des illustrations, parfois complexes, qui multiplient les significations. Elle découvre le langage scriptovisuel bien avant la presse et ne se parcourt pas de façon linéaire. Elle doit être lue, il faut arrêter sa marche pour la déchiffrer et l'interpréter. Mais elle se distingue du journal parce qu'elle parle à tous, partisans, indécis, et même adversaires. Les passants, s'ils ne lisent qu'une gazette, peuvent voir toutes les affiches, et sont donc confrontés à des arguments contradictoires. Un abbé militant, É. Fourié, l'observe au début du vingtième siècle : "Le journal peut être comparé à une réunion privée où des amis réunis entre eux pardonneront quelques écarts à l'orateur qui leur parle. L'affiche rappelle la réunion publique, où la moindre maladresse commise par l'orateur soulèvera la salle entière contre lui et servira de prétexte au tapage des adversaires"l.

\section{La presse industrielle se tient à l'écart}

La presse d'opinion tient le haut du pavé lorsque le journal, artisanal, voit sa production forcément limitée par la faiblesse des possibilités techniques qu'offrent les presses en bois et l'assemblage manuel des caractères. Dans la seconde moitié du XIX ${ }^{\mathrm{e}}$ siècle, quand apparaissent les rotatives, puis les linotypes, les tirages peuvent passer de milliers à des centaines de milliers, voire à des millions d'exemplaires. La presse devient industrielle et ce sont maintenant des brasseurs d'affaires plutôt que des brasseurs d'idées, des entrepreneurs plutôt que des politiques, qui vont investir dans la presse. Polydore Millaud est spécialiste en affaires financières avant de créer Le Petit Journal à Paris ; Émile Rossel, qui lance Le Soir à Bruxelles, se préoccupe surtout de publicité, tout comme Rudolph Mosse, créateur du Berliner Tageblatt; Leopold Ullstein, qui introduit à Berlin le type de la Boulevard Presse, a été d'abord propriétaire de papeteries.

Le journal industriel ne peut plus se limiter à l'audience réduite de groupes de partisans, il doit se lancer à la conquête du grand public. Son prix diminue, son contenu évolue. Les deux choses vont de pair, et Rossel l'a bien compris, annonçant que son quotidien sera

Fr. HeInderyckX et G. ThOveron, Un siècle d'affiches politiques en Belgique francophone, Liège, CÉFAL, 2003.

I De l'affichage politique, Paris, Maison de la Bonne Presse, 1902, p. 2. 
tout à la fois "sans couleur politique et absolument gratuit (...) En observant une neutralité absolue, il entend ne jamais blesser ni heurter l'opinion de personne". Heurter un lecteur, c'est risquer de le perdre. Prendre trop franchement parti, c'est se tenir à distance d'une bonne part du public potentiel.

La preuve en sera faite lorsque des milliers de lecteurs quitteront Le Petit Journal pour Le Petit Parisien, tous deux quotidiens à un sou : le premier, sous la plume d'Ernest Judet, bataillait furieusement contre Dreyfus, irritant tout autant les partisans de ce dernier que les lecteurs que cette querelle lassait. Le second pratiquait un prudent attentisme.

Au fur et à mesure que baisse la passion politique, on voit se réduire la clientèle potentielle des gazettes de combat. Cette clientèle, dira Jacques Kayser, "réclame un journal conçu pour elle, c'est-à-dire à rayonnement limité, dont l'existence est incertaine et toujours menacée, véritable anachronisme au sein d'une évolution économique qui tend au gigantisme"'. Et il cite un vétéran du reportage politique américain, Charles B. Cheney, pour qui “ce n'est plus l'usage de combattre vigoureusement lors d'une campagne politique. En fin de compte, cela ne paie pas de se faire tant d'ennemis".

L'objectivité, qui respecte les valeurs de chacun, devient la règle d'or. Le Soir, précise dans ses colonnes son fondateur, "se bornera à relater les faits importants, laissant à l'intelligence du lecteur le soin d'en tirer des conclusions". Il ne s'agit plus de dire au client ce qu'il doit penser, mais de lui donner les éléments propres à lui permettre de penser par lui-même. Ou de lui en donner l'impression.

Le passage de la presse d'information à la presse d'opinion s'opère d'autant plus aisément que les grandes luttes entre fractions de la bourgeoisie s'estompent et qu'il faut faire face à la montée du mouvement ouvrier : le grand journal de nouvelles contribue à détourner le public populaire des feuilles socialistes.

Le quotidien se fait plus contemplatif que combatif. Il continue à avoir des idées, mais des idées moyennes, point trop tranchées ni même explicites, et qui peuvent fluctuer en s'efforçant de s'adapter aux caprices de l'opinion dominante. S'il éprouve le besoin de faire campagne, il mènera son combat insidieusement. "Dans le passé, observe Louis Gabriel-Robinet, c'est la passion, la lutte, opposant les plus grands lutteurs. Dans le présent, c'est la technique qui donne

1 Mort d'une liberté, Paris, Plan, 1955, p. 98. 
l'apparence de l'objectivité et n'est trop souvent qu'un déguisement

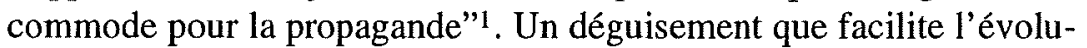
tion du langage graphique, scriptovisuel, et qui fait que, selon l'expression de Lucien Romier, "l'opinion ne se fait plus par la réflexion, elle se fait par les yeux (...) l'opinion commune apprécie l'importance des événements d'après la mise en page et la composition typographique"2.

Commentaires et nouvelles politiques voient d'ailleurs leur importance diminuer en même temps que s'élargit le champ de l'information, géographiquement grâce au télégraphe, socialement avec la diversification qui accompagne l'accroissement du lectorat. De nouvelles rubriques, du sport aux faits divers, sont appelées à répondre aux attentes de nouveaux lecteurs. Le journal, dont le format et la pagination grandissent, ne va plus se lire, comme à ses débuts, de la première à la dernière ligne, comme un livre; la lecture fractionnée devient la règle et l'on saute plus volontiers les commentaires que les nouvelles.

À l'éditorial politique se substitue donc souvent la chronique, qui parle de tout et de rien; l'un et l'autre occupent toujours la place d'honneur, en haut à gauche de la Une, c'est la partie noble du journal et il convient de l'afficher, même si l'on est conscient que le lecteur n'y accorde plus guère d'attention. La grande nouvelle du jour est rejetée à droite, on compense cette moins bonne place par un titre en gros caractères.

Les quotidiens engagés, ceux qui conservent une étiquette (ce sera très longtemps le cas de la plupart des journaux belges) assurent que, si leurs commentaires sont orientés, leur information est aussi objective que celles des quotidiens indépendants. Il est diffícile de jouer les deux rôles en même temps, tenir boutique et tenir le front des idées, et c'est cependant le pari que tiendra la famille fondatrice de $L a$ Libre Belgique, réussissant à faire un journal où l'esprit partisan n'altérait pas la qualité de l'information, où pendant longtemps un changement de caractères indiquait au lecteur que l'on passait de l'énoncé objectif au commentaire engagé.

Il est d'ailleurs possible d'offrir aux lecteurs un choix d'opinions. Des journaux vont découvrir les vertus du pluralisme : il n'est pas nécessaire que chaque tendance politique soit tenue de

1 L. GABRIEL-RoBinet, Journaux et journalistes, Paris, Hachette, 1962, p. 205.

2 L. ROMier, Explication de notre temps, Paris, Grasset, 1925, p. 139. 
disposer de sa publication porte-parole si toutes les idées -ou en tout cas beaucoup d'entre elles- sont présentes dans un même support. C'est déjà l'expérience, mais qui ne fut pas d'abord comprise, tentée par Le Matin de Paris : à ses débuts, en 1884, il publiait en alternance les articles de tête de deux chroniqueurs de gauche et de deux chroniqueurs de droite. Plus tard, dans l'entre-deux-guerres, Paris Soir et Le Soir à Bruxelles ouvriront des Tribunes libres où des partis trouvent à s'exprimer. Des partis, mais non tous les partis, il ne s'agit que de présenter les opinions convenables. Le Soir ne s'ouvre qu'aux partis traditionnels, Paris Soir exclut monarchistes et communistes, sur le modèle d'ailleurs de la radio française naissante, où "seuls les partis au pouvoir ou ceux qui y étaient auparavant ou qui y seront après les prochaines élections ont le droit d'être cités ou commentés"'.

Le débat est donc bridé, puisque les opinions extrêmes ne participent pas à la confrontation : n'y on droit que des partis modérés, habitués des gouvernements et de leurs compromis. Un débat qui se tient d'autant plus en sourdine qu'il n'est évidemment pas convenable d'abuser de l'hospitalité du média dont vous êtes l'invité, ni habile de heurter trop frontalement les idées de lecteurs -ou d'auditeurs- qui sont loin de partager les points de vue auxquels ils se trouvent ainsi confrontés.

Ces lecteurs, le journal leur dit surtout ce qu'ils peuvent penser - ou peut-être ce qu'il ne doivent pas penser s'ils veulent éviter de se retrouver isolés de l'opinion publique, telle que la définira plus tard Elisabeth Noëlle-Neuman. Selon sa théorie de la spirale du silence (Schweigespirale), la plupart des gens ne veulent pas se marginaliser en allant à l'encontre du climat d'opinion ambiant, et préfèrent se taire plutôt que de courir le risque d'un conflit avec leur entourage -l'opinion publique, c'est alors celle qui "peut être exprimée en public sans risque de sanctions, et sur laquelle peut s'appuyer l'action menée en public"2. L'opinion qu' on ne partage pas toujours, mais qu'on ne contredira pas sans précaution. Un climat dominant que l'on peut percevoir dans son environnement, mais que répercutent et précisent les médias. Voilà pourquoi ces derniers sont aujourd'hui si friands de sondages qui semblent leur dire voilà ce qui se pense, voilà d'ailleurs sans doute ce que vos lecteurs pensent.

1 C. BRochand, Histoire générale de la radio et de la télévision en France, tome I : 1921-1944, Paris, La Documentation française, 1994, p. 450.

2 "The Spiral of Silence", Journal of Communication, $\mathrm{n}^{\circ} 24$, 1974, pp. 43-54. Traduit dans Hermès, Paris, ${ }^{\circ}$ 4, pp. 181-189. 
L'opinion franchement affichée ne reprend de l'importance que dans les périodes de tension politique où la presque totalité du public se sent impliquée, par exemple en Belgique l'affaire royale qui verra Le Soir prendre parti contre Léopold III, ou en France le Front populaire : "Dans son ensemble, la presse française atteint en 1936 un degré extrême de «politisation». De la première à la dernière ligne, la plupart des quotidiens sont remplis de politique (...) divers périodiques, qui, en temps ordinaire, se soucient peu de politique, se font largement l'écho des préoccupations générales "', parfois avec une violence extrême : accusé par l'hebdomadaire Gringoire d'avoir déserté pendant la guerre, le ministre de l'Intérieur, Roger Salengro, pourtant mis hors de cause par la Chambre, est conduit au suicide.

Les familles d'idées qui souhaitent continuer à agir directement sur l'opinion ne peuvent maintenir de fidèles organes de presse qu'en en assurant le financement : ces journaux sont d'organisation plutôt que d'opinion. Alors que l'Europe est confrontée à la montée de partis totalitaires, staliniens ou fascistes, pour qui la politique est religion, la propagande se substitue au débat, les symboles aux arguments. Il s'agit de diffuser puis nourrir, au-delà des positions du parti et de la discipline qui doit régner en son sein, une foi, l'espoir en des lendemains radieux, en un monde (un ordre) nouveau. D'inculquer des dogmes qui permettent d'escamoter le débat -le bien ne peut se confronter au mal, ce serait le reconnaître et pactiser avec lui- et de créer et entretenir des réflexes conditionnés. Les adversaires sont démasqués, dénoncés, l'injure refleurit, les slogans simples se substituent aux arguments.

\section{La télévision met en scène}

La fin de la seconde guerre mondiale ouvre un temps où certains croient pouvoir sonner le glas des idéologies. C. Wright Mills publie en 1956 The Power Elite, où il montre que la chose publique est devenue l'affaire d'un groupe de spécialistes au sein duquel, et audelà des clivages politiques, finit par s'installer une certaine unité de vue. En 1960, Daniel Bell annonce The End of Ideology. Dans ce contexte, la propagande n'a plus de sens, et le débat tend à se limiter à des problèmes techniques de gestion.

1 L. Bodin et J. Touchard, Front populaire 1936, Paris, A. Colin, coll. "Kiosque", 1961, p. 229. 
Les pays démocratiques entrent dans l'ère de la communication politique ${ }^{1}$, née du mariage de la télévision, des sondages et des techniques publicitaires. L'essentiel, pour un parti ou un candidat, est d'élargir sa part de marché électorale en offrant une bonne image, répondant à l'idée que se font les électeurs de ceux qui sont le mieux capables de diriger la société. Des citoyens qui se retrouvent spectateurs plutôt qu' acteurs de la chose politique.

La télévision donne une dimension nouvelle à l'information quotidienne, qu'elle met en scène. Elle fait de la réalité un spectacle où le public cherche un divertissement. En Europe, où elle est d'abord service public et tenue en principe à une stricte objectivité, elle réalise vite que l'information sur les opinions est plus spectaculaire en débats qu'en tribunes.

La chose politique prend une dimension ludique. Kurt et Gladys Lang citeront un exemple aujourd'hui vieux d'un demi-siècle, celui des discours télévisés du sénateur Everett Dickson lors de la Convention républicaine de 1952, discours qui furent savourés comme une performance de premier ordre plutôt que comme des interventions aux conséquences politiques ${ }^{2}$. Les fameux débats KennedyNixon de 1960 seront surtout perçus comme une dramatique confrontation entre deux hommes, les téléspectateurs, démocrates comme républicains, ayant pour la plupart oublié les arguments échangés et se posant surtout la question : "Qui a gagné ?". Certains pensèrent même que les participants à ce Quiz, les deux candidats à la présidentielle, étaient payés pour leur prestation ${ }^{3}$ : “...un grand nombre de messages d'information, note alors Holde Lhoest, quelle que soit l'intention de l'émetteur, peuvent être perçus en tant que faits essentiellement divertissants destinés à renforcer le plaisir de communication immédiat et détachés de tout contexte normatif"4.

Pour les débats désormais télévisés, cela va se traduire par une perte du fond au profit de la forme. Cette évolution se lit dans

1 J'ai montré les étapes de la mue des médias (opinion, information, spectacle) dans Le troisième âge du quatrième pouvoir, Bruxelles, Labor, 1999 et de celle des techniques de persuasion (débat, propagande, communication) dans $L a$ marchandisation de la politique, idem, 2003.

2 Politics and Television, Chicago, Quadrangle Books, 1968, pp. 193-194.

3 E. Katz, "Les débats Kennedy-Nixon", in Études de radiotélévision, Bruxelles, RTB, n 2, 1963, pp. 3-1.

4 "Le divertissement, fonction psychosociale de l'information télévisée", in Publics et techniques de la diffusion collective, études offertes à Roger Clausse, Bruxelles, ULB, 1971, pp. 208-209. 
l'histoire générale qu'en trace Noël $\mathrm{Nel}^{1}$, mettant en évidence quatre étapes qui se déterminent en fonction du modèle qui les domine : naissance du genre, 1960-68, grandes controverses politiques, 196874 , débats culturels ou de société 1974-81, période du spectaculaire 1981-86. Cette étude est publiée en 1988, et le spectacle, depuis, n'a pas cessé de prendre de l'importance, au fur et à mesure que la dérégulation du marché transformait la télévision. Autrefois, les images de celle-ci s'adressaient plutôt, comme, le notent Charaudeau \& Ghiglione, à "une cible de téléspectateurs relativement bien équilitrés quant à leur intellect et à leur affect", avec des émissions bien délimitées où l'on évitait de mélanger les genres, et non sans ambitions pédagogiques. Aujourd'hui, elles valorisent ceux dont "l'intellect est troublé et l'affect envahissant" 2 . Elles les valorisent, et les flattent aussi, en leur ouvrant ses portes -la télé dite réalité est la forme extrême et presque caricaturale de cette tendance. Elles leur donnent une place dans les émissions: depuis 1970, observe Sébastien Rouquette, les débats de société ont accordé moins d'importance aux experts pour ne laisser "en moyenne, pas moins de la moitié de l'ensemble des places disponibles aux membres de la société civile. Les progressions ultérieures ne proviennent pas d'une extension de cette place. Elles s'expliquent plus modestement par la disparition des derniers responsables d'émissions totalement réfractaires à l'idée d'autoriser les Français moyens à intervenir dans leurs programmes"3. Des Français moyens, des témoins, plutôt que des experts, des messieurs-tout-le-monde plutôt que des personnalités appartenant à des organisations représentatives, de simples citoyens plutôt que des politiques. "En ayant dénié aux représentants du peuple le droit d'être des porte-parole, c'est-à-dire tout à la fois ceux dont la parole est autorisée et fait autorité, le citoyen ordinaire -aidé par la télévisions'autodésignerait comme porteur d'une parole légitime qu'il peut exhiber sur l'écran dont l'audience redouble la légitimité"4. Parole qui est aussi la nôtre, puisque nous pouvons nous identifier à l'un ou l'autre des personnages quelconques ainsi autorisés à s'exprimer.

1 N. NEL, A fleurets mouchetés : 25 ans de débat télévisé, Paris, INA-La Documentation française, 1988.

2 P. Charaudeau et R. Ghiglione, La parole confisquée, Paris, Dunod, 1997, pp. 68.

3 S. RouquetTe, Vie et mort des débats télévisés 1958-2000, Bruxelles, INA-De Boeck, 2002, p. 94.

4 P. Charaudeau et R. Ghiglone, op. cit., p. 170. 
L'explosion du talk-show, qui s'efforce de monter un conflit en spectacle, de mettre une joute en scène, résulte de cette évolution. Charaudeau et Ghiglione ont participé à une équipe internationale analysant les émissions Ciel! mon mardi, dans une sorte d'arène, $L a$ Vida en un xip, dans un amphithéâtre, et Maurizio Constanzo Show, sur une scène de théâtre; ils montrent leurs différences -mais aussi ce qu'ils ont en commun: "Le projet ludique du talk-show français de mise en spectacle d'une parole conflictuelle, correspondant à un imaginaire du jouir de la polémique; le projet éthique du talk show catalan de mise en scène d'une parole domestiquée, correspondant à un imaginaire du conflit entre passion et raison; le projet d'une esthétique baroque du talk show italien d'une mise en scène d'une parole flottante, correspondant à un imaginaire du jouir de la théâtralité de la vie. Tous les trois, pourtant, malgré leurs différences, participent d'un même simulacre, celui qui fait croire que ces genres d'émissions contribuent au développement du débat démocratique dans l'espace public"l.

Il s'agit de démagogie plutôt que de démocratie. "Il n'existe pas un espace ouvert à tous ceux qui le veulent, mais des agents qui décident, en fonction des lois propres de fonctionnement du champ journalistique, ce qui mérite ou non d'être porté à la connaissance de publics plus ou moins larges et hétérogènes socialement"2. Ces pratiques traduisent une prise de pouvoir par les présentateurs. Ceuxci sont convaincus d'être les seuls à savoir ce qui fera de la bonne télévision, c'est-à-dire du spectacle. S'ils ne peuvent éviter la présence d'hommes et de femmes représentatifs, et notamment de politiques, sur le plateau, ils s'attachent, comme le dimanche midi sur RTL-TVi ou la RTBF, à en multiplier le nombre. Le temps d'intervention de chacun est réduit et la domination s'affirme de celui qui dirige et oriente les échanges. Comme le note Serge Halimi dans une comparaison éclairante, "tel le journaliste politologue choisissant les questions qu'il se pose, qu'il posera et auxquelles les sondés devront répondre, le journaliste présentateur ordonnance à son gré le monde des idées, l'identité et le nombre des intervenants, l'opportunité de leur donner la parole, le moment et les règles de la

1 Ibid., pp. 158-159.

2 P. Champagne, Faire l'opinion, le nowveau jeu politique, Paris, Éd. de Minuit, 1990, p. 243. 
rencontre"l. Sondages et interviews déterminent les thèmes dont il faut parler, établissant ainsi l'ordre du jour, l'agenda de la vie politique $^{2}$.

Le rôle des téléspectateurs invités aujourd'hui à intervenir dans les émissions consacrées aux affaires publiques semble être d'ouvrir les uns avec les autres, ou avec les politiques, un débat que ces derniers renoncent de plus en plus à entamer entre eux. Des émissions comme Lieu public, à laquelle la RTBF a décidé de renoncer, ou hier Droit de réponse (dont le maître d'œuvre, Michel Polac, disait "on me dira ce qu'on voudra de mon émission, qu'elle est brouillonne, énervante, insaisissable mais, moi, j'affirme que chaque numéro comporte cinq minutes d'insolite, d'original, qu' on ne trouve pas ailleurs"), ces émissions donnent lieu à une agitation que les politiques tentent aujourd'hui de fuir.

Les journalistes savent qu'il est devenu difficile de créer une tension dramatique dans une émission politique. C'était une vaine tentative que celle de Paul Amar offrant des gants de boxe à JeanMarie Le Pen et Bernard Tapie, dont il était censé, le 3 juin 1994, sur France 2, modérer le débat. Son initiative lui valut une sanction, il tenta de se justifier en assurant qu'il ne voulait qu'exorciser la violence annoncée de cette rencontre. Une violence annoncée, mais qui ne se produisit point: le choc entre les deux bateleurs fut bien moins rude que lors de leur précédente rencontre. C'est que les politiques se montrent désormais sensibles aux avis des conseillers en communication qui, observe Roland Cayrol, "ont tendance à gommer les aspérités, à enlever «tout ce qui dépasse», tout ce qui pourrait choquer une catégorie, tout ce qui pourrait faire perdre des voix, et à modérer les positions en toute chose"3. Et qui aussi, conscients de ce que le public se montre plus perméable aux personnalités qu'aux idées, considèrent qu'un candidat suscitera davantage l'adhésion s'il refoule son agressivité. Des débats Mitterrand-Giscard $(1974,1981)$ aux débats Mitterrand-Chirac (1988) et Chirac-Jospin (1975), la baisse de tension est évidente. C'est, note Cayrol, qu'il "faut renoncer au discours politique (...) renoncer aux interruptions qui vous

1 S. Halimi, "Ces débats médiatiquement corrects", Manière de voir, $\mathrm{n}^{\circ} 46$, Paris, juillet-août 1999, p. 91.

2 Sur la fonction d'agenda, voir par exemple E.F. SHAw, "Agenda-Setting and Mass Communication Theory", Gazette, La Haye, vol. XXV, 2, 1979, pp. 96-105.

3 R. CAYrol, Médias et démocratie : la dérive, Paris, Presses de Sciences Po, 1997, p. 53 . 
démangent, être décidément «gentil», «cool». Il faut, en un mot, parler télévision, comme le font «les gens de la télé». (...) On est dans un monde où la volonté de séduction, disons le racolage, remplace l'essai de persuasion"'.

Les politiques préfèrent se montrer là où ils pourront déployer leur personnalité plutôt que leur projet politique, s'afficher sans contradicteur, et sans être embarrassés par des questions dérangeantes, des émissions de divertissement comme le Vivement Dimanche de Michel Drucker sur France 2 ou les @llumés.be de la RTBF. Au point que les émissions électorales vont tendre, elles aussi, à fournir du plaisir aux téléspectateurs et, aux politiques, des occasions de valoriser leur image.

On le verra à l'occasion des législatives belges de 2003. Sur RTL, Élections 2003 est un parcours ludique, une course minutée où il faut savoir répondre vite aux questions ; sur la RTBF, dans C'est ma voix, les candidats, dont la voix est transformée, s'affrontent sans se voir, ignorent leurs identités et même leurs partis respectifs; sur Canal+, dans Nom de nom, l'animateur semble mettre en œuvre un test de projection.

Les journalistes politiques appelés à commenter l'actualité ont repris le rôle des éditorialistes de jadis, mais, tenus de sembler aussi objectifs que les médias qui les emploient, leurs propos oscillent autour d'une sorte de pensée unique et politiquement correcte : “...quand l'orthodoxie s'exprime", observe Halimi, "elle le fait sans vis-à-vis. Juge-t-on jamais qu' Alain Duhamel ou Jean-Marc Sylvestre défendent des points de vue «polémiques»? Oppose-t-on à chacun de leurs éditoriaux quotidiens la réfutation d'un adversaire ?"2. Bien sûr que non.

La presse écrite cherche à s'engager dans les voies ouvertes par la télévision, et si elle ne dispose pas des mêmes moyens de spectacularisation, participe elle aussi, du journalisme magazine, non plus magasin à rayons multiples, mais magasin où tous les produits sont présentés en vrac, magazine qui a gommé "les frontières entre les types de discours (...) entre reportage et commentaire, entre information et divertissement, entre réalité et fiction"3 selon

1 Ibid., p. 59.

2 S. HALIMI, op. cit., p. 92.

3 J. Charron et J. DE Bonville, "Le paradigme du journalisme de communication : essai de définition", Communication, Québec, Univ. Laval, déc. 1996, vol. 17, n² 2 , pp. 51-97. 
l'expression de Charron et Bonville (qui nomment cela journalisme de communication, "forme de journalisme associée à une société très fortement urbanisée et dont la structure économique s'appuie sur la consommation intensive et sur les activités du secteur tertiaire, particulièrement de loisir", mais si peu sur la politique.) Dans ce contexte, le débat, la polémique, sont devenus des contenus parmi d'autres, trouvant leur place dans des rubriques diverses, comme la caricature, des dessins de Plantu ou Kroll aux Guignols de l'info, promus au rang d'éditorialistes. 